\title{
Le pouvoir des mots : émission et réception du discours du banquier central
}

Mickaël Clévenot, Ludovic Desmedt et Matthieu Llorca

\section{(2) OpenEdition \\ Journals}

Édition électronique

URL : http://journals.openedition.org/ei/1016

DOI : 10.4000/ei.1016

ISSN : 2553-1891

Éditeur

Association Économie et Institutions

Référence électronique

Mickaël Clévenot, Ludovic Desmedt et Matthieu Llorca, « Le pouvoir des mots : émission et réception du discours du banquier central », Économie et institutions [En ligne], 22 | 2015, mis en ligne le 01 juin 2015, consulté le 19 avril 2019. URL : http://journals.openedition.org/ei/1016 ; DOI : 10.4000/ei.1016

Ce document a été généré automatiquement le 19 avril 2019

Revue Économie et institutions 


\title{
Le pouvoir des mots : émission et réception du discours du banquier central
}

\author{
Mickaël Clévenot, Ludovic Desmedt et Matthieu Llorca
}

Nous remercions vivement les participants du colloque "Crise de l'euro, effets discursifs et changements institutionnels : approches transdisciplinaires ", Université Picardie-Jules Verne, particulièrement J. Marques-Pereira, ainsi que les deux rapporteurs anonymes de cette revue pour leurs remarques, commentaires et suggestions. Des discussions avec F. Hervé, M. Zouaoui puis $P$. Steiner ont permis d'enrichir la réflexion. Nous restons responsables des insuffisances de ce texte. Une version antérieure est consultable : https://hal.archives-ouvertes.fr/hal-01089003

1 Dans les médias, la parole des banquiers centraux a été récemment parée de pouvoirs impressionnants: la manière dont sont désormais attendues et décryptées les déclarations de Mario Draghi, Janet Yellen ou Haruhiko Kuroda incite à penser que les dirigeants de la B.C.E., du Fed ou de la Bank of Japan se sont transformés en « oracles » omnipotents (voir Rogoff, 2014). Toute inflexion de leur discours, l'utilisation de tel mot plutôt que tel autre ou l'expression d'un jugement sur l'avenir tiendraient désormais lieu de politique monétaire. Or, depuis quelques années, deux champs disciplinaires d'ordinaire distants, la théorie économique «standard», d'une part, et la sociolinguistique, d'autre part, questionnent le rapport existant entre les discours et la réalité. Dans le contexte actuel de crise et de reformulation des politiques monétaires, ce questionnement s'applique particulièrement aux Banques Centrales, institutions devenues particulièrement actives dans les domaines de la communication. Il y a peu, Lebaron notait encore que «Le discours d'un banquier central en période de crise sociale peut sembler anecdotique si on le compare aux interventions des acteurs politiques et médiatiques dominants, et il paraît en effet particulièrement opaque à première vue. » (Lebaron, 2006, p. 8). Le contexte financier actuel a bouleversé cette vision.

2 Sur le versant économique, les travaux de Blinder (2004, 2008, 2009), Mishkin (2004), Gerlach (2004, 2007), Issing (2005), Rosa et Verga (2005, 2007), Woodford (2005) ou Jansen 
(2011) ont justifié une politique de communication active de la part des instituts d'émission. Un consensus a émergé, d'abord sur le plan théorique, puis pratique, considérant que l'information détenue par la Banque centrale devait être largement diffusée, de manière à ancrer et guider les anticipations des acteurs économiques. Sur le versant sociologique, les travaux de Krippner (2007), Aitken (2007), Hall (2008), Brasset et Clarke (2012), Clarke et Roberts (2014) ou Velthuis (2015) ont souligné les inflexions du " bank talk» (Karl, 2013). Le travail récent de Holmes (Economy of words, 2014), tente la conciliation et conjugue l'argumentaire sociologique et économique pour étudier les transformations dans la rhétorique utilisée par les instituts d'émission.

3 La plupart des travaux en question visent à interroger la performativité de ces discours. Si l'on suit Callon et Muniesa, de manière générale, «En linguistique, on dit d'un énoncé qu'il est performatif quand il instaure ce dont il parle " (Callon, Muniesa, 2008, p. 1). En simplifiant, il existe deux grandes approches de la performativité : la plus ancienne, issue des travaux d'Austin (1962) s'interroge sur les liens entre mots et actions et privilégie l'étude des « actes de langage » (Searle, 1972, 1998). Cette approche « austinienne » insiste sur les énoncés qui, du fait de leur énonciation, accomplissent un acte ${ }^{1}$. La vision plus récente, développée en particulier par $\mathrm{M}$. Callon et $\mathrm{F}$. Muniesa, insiste sur les « agencements socio-techniques » nécessaires à la performation du monde. Dans ce cadre, c'est en particulier l'impact des énoncés théoriques qui est étudié : "cette intuition permet de qualifier les situations dans lesquelles l'objet sur lequel porte un travail scientifique n'est pas simplement constaté ou décrit, mais modifié, voire appelé à exister.» (Callon, Muniesa, 2008, p.1). En effet, les sciences sociales produisent des discours qui peuvent transformer une partie de la réalité ${ }^{2}$.

Lorsque l'on étudie l'impact des discours des banquiers centraux sur le monde, ces deux approches peuvent être mobilisées. Dans la lignée de Brisset (2012), on peut qualifier la logique économique de "stratégique » et la logique sociologique d'« interprétative ». Il est alors possible de se pencher sur la façon dont la théorie a mis en exergue le banquier central pour " performer » le monde ${ }^{3}$, d'une part, et sur l'influence son discours sur les représentations des agents, d'autre part. Alors qu'historiquement les banquiers centraux étaient caractérisés par leur mutisme, l'accroissement du volume de discours émis a été préparé par une théorisation de la politique économique inspirée de la théorie des jeux. À partir du phénomène d'incohérence temporelle mis en avant par Kydland et Prescott (1977) et d'un biais inflationniste repérable chez les décideurs (Backus et Driffil, 1985), les théoriciens ont favorisé la mise en place de banquiers centraux autonomes vis-à-vis du pouvoir politique, de manière à crédibiliser les politiques anti-inflationnistes. L'approche en termes d'agencement (Callon) convient pour comprendre cette mutation stratégique. D'autre part, l'aspect interprétatif s'intéresse aux actes de langage : dans ce travail nous nous intéresserons en particulier à l'éventail communicationnel déployé par la dernièrenée des grandes banques centrales, la BCE. À titre d'illustration, nous proposons de mesurer la tonalité des éditoriaux du bulletin mensuel de la BCE . Pour ce faire, nous nous inspirons de la méthode de traitement textuel du Beige Book de la Fed développé par les économistes Sadique, In, Veeraraghavan, et Wachtel (2013). Ces auteurs caractérisent la tonalité du rapport puis étudient son contenu prédictif en utilisant un logiciel d'analyse textuelle. Notre étude porte sur les éditoriaux de la BCE entre 1999 et 2014 et indique un « tournant lexical ${ }^{5}$ lors de la crise des subprimes : la tonalité des mots varie à partir de ce moment. Nous nous intéressons ensuite à un moment critique de la période récente, en juillet 2012, lorsque M. Draghi annonça que « dans le cadre de son mandat, la BCE est déjà 
prête à tout faire pour préserver l'euro, et croyez-moi, cela sera suffisant $»^{6}$. Malgré l'absence de décision formelle à ce sujet, cette prise parole atténua les tensions sur les marchés obligataires: dans une ère de politiques non conventionnelles et de crise de l'euro, on s'accorda à penser que ces mots modifièrent le monde financier. Pourtant, cette analyse des discours de la BCE, si elle indique les inflexions du discours et les ruptures de ton, montre les limites des études purement communicationnelles. Il nous semble que pour sonder la qualité performative des «dits et écrits » des banquiers centraux, les apports de l'analyse institutionnelle sont nécessaires.

5 La lecture des analyses consacrées à la communication des instituts d'émission fait apparaître que la littérature économique (qui amplifie le rôle du banquier central) comme les approches sociologiques (qui étudient ses « actes de langage ») reposent sur une vision très schématique $d u$ "public » vers lequel sont orientés les discours. Une définition précise de l'audience visée constitue un point aveugle de ces approches. Afin de préciser ce thème, nous mettons à contribution certaines des intuitions de Bourdieu lorsqu'il aborde la question du langage. Il insistait surtout sur les relations de pouvoir sousjacentes : » l'échange linguistique est aussi un échange économique, qui s'établit dans un certain rapport de forces symbolique entre un producteur, pourvu d'un certain capital linguistique, et un consommateur (ou un marché), et qui est propre à procurer un certain profil matériel ou symbolique. » (Bourdieu, 2001, p. 99)7 . De fait, ce sont ces rapports entre l'émetteur du discours et ses "consommateurs" qui sont particulièrement intéressants à étudier. Il nous semble que cette voie est compatible avec certaines théories économiques de type institutionnaliste. En s'intéressant de près à cette instance particulière que sont les marchés financiers, les approches en termes de conventions et de régulation surmontent certaines apories de l'analyse économique standard comme de certaines études sociologiques.

\section{Théorie économique et central banking: indépendance, crédibilité, transparence}

Dans un court texte intitulé «The triumph of central banking?», Paul Volcker évoquait la possibilité pour les banquiers centraux de devenir un jour des "héros populaires modernes" (Volcker, 1990, p. 3). Pourtant, sur le long terme, les Banques centrales ont été caractérisées par un ensemble de décisions techniques, l'explicitation de ces actes étant rejeté en dehors du périmètre de leurs missions. Le laconisme des décideurs en matière de politique monétaire devint ainsi au XXe siècle une de leurs caractéristiques centrales. Issing (2005) rapporte un débat pendant l'entre-deux guerres entre J.M. Keynes, membre du Comité Mc Millan, et Sir Ernest Harvey, Deputy Governor de la Banque d'Angleterre. Aux demandes de Keynes sur la logique et l'explicitation de certaines décisions, Harvey répondit qu'il était "dangereux " d'être trop clair... ${ }^{8}$ Décrivant une situation plus contemporaine, Harris écrit que : "Paul Volcker, a devote cigar-smoker and Fed chair from 1979 to 1987, would literarily and figuratively blow smoke at congressional hearings, baffling congressmen with complex economic arguments. Greenspan continued this tradition with "Fedspeak"-speeches with hidden code words that only experienced Fed watchers could understand. Only with great reluctance did Greenspan become more open during the second half of his term." (Harris, 2008, p. 6). 
7 Ainsi, la vision que l'on pouvait avoir de la politique de communication des Banques centrales était celle d'un domaine crypté, entouré de mystères (Brunner, 1981). Peu de déclarations émanaient de la part des banquiers centraux, une codification extrême était de mise. En conséquence, la sobriété des locuteurs pouvait confiner à l'« ésotérisme »'. De fait, au cours du mandat de Greenspan à la tête du Fed, les banquiers centraux devinrent plus diserts sur leurs motivations et leurs objectifs. Alan Blinder fut ainsi un des premiers à se demander si la communication des Banques Centrales importait vraiment; à l'époque, il répondait que "jusqu'à présent, il n'y a pas eu de recherche pour conclure » (Blinder et al., 2001, p. 9). Or, d'un point de vue académique (à travers une littérature abondante) et pratique, la communication des instituts d'émission est devenue une préoccupation majeure au cours de ces quinze dernières années. Cette dimension nouvelle a été intégrée à la conduite et à l'orientation de la politique monétaire (Woodford, 2003).

8 Un tel revirement s'inscrit dans le débat théorique à propos de l'efficacité des politiques budgétaire et monétaire sur la stabilisation macroéconomique : l'arme budgétaire a été progressivement discréditée et on considère que la politique monétaire jouerait seule un rôle significatif. Selon une opinion répandue (Woodford, 2003), la production de prévisions, d'informations et un discours de la Banque centrale expliquant ses objectifs et les moyens d'y parvenir ancreraient de manière satisfaisante les anticipations des agents.

\subsection{Politique monétaire et anticipations : vers le consensus}

Les fondements théoriques de la révolution dans la communication des Banques Centrales peuvent être trouvés dans le corpus des "nouveaux classiques». Ces auteurs se sont intéressés au cours des années 1980 à l'impact des discours de politique économique sur les anticipations du public. Dans le cadre théorique des anticipations rationnelles, et dans une démarche qui insistait sur les relations entre " autorités » et "public », le souci de la cohérence devait conduire à ne pas tromper les agents (Kydland et Prescott, 1977). Dans le cas contraire, les autorités seraient confrontées au phénomène d'incohérence temporelle, corrélée à un biais inflationniste repérable chez les décideurs ${ }^{10}$. Dans ce modèle, «pour qu'une mesure de politique économique annoncée pour le futur soit prise au sérieux par les agents privés, il faut que ceux-ci soient certains que la question «le gouvernement aura-t-il intérêt à mettre en œuvre demain ce qu'il annonce aujourd'hui ?» recevra une réponse positive.» (De Vroey, Malgrange, 2007, p. 19). Rapidement, la question de la politique monétaire fut traitée de manière spécifique (Barro et Gordon, 1983). Dans ce cadre d'analyse, un gouvernement ne peut mener une politique de lutte contre l'inflation que s'il est jugé crédible. Le gouvernement ayant un horizon temporel réduit sera toujours tenté de se servir de la politique monétaire afin de réaliser un arbitrage chômage inflation qui conduira immanquablement à une augmentation de l'inflation. La crédibilité ne peut donc être assurée que par une instance d'émission monétaire indépendante. Pour Barro et Gordon, on réduirait ainsi l'effet des groupes de pression pouvant influencer les décisions techniques concernant la monnaie (et amener à des choix inflationnistes).

10 Dès 1985 - alors même que les banquiers centraux sont encore caractérisés par leur mutisme-, Backus et Drifill (1985) s'interrogent sur le degré de véracité que l'on peut attribuer aux annonces d'un gouvernement dans sa lutte contre l'inflation. Ces deux auteurs adoptent un raisonnement en termes de théorie des jeux et insistent sur le critère 
de réputation, en distinguant entre les gouvernements " hard nosed » et les "wet », selon qu'ils seraient plus ou moins tolérants vis-à-vis de l'inflation. On classera plus tard les gouvernants en «faucon»(hawks), partisans d'une politique monétaire moins accommodante et à l'opposé en « colombe » (doves). L'article de 1985 affirme que la parole politique en matière monétaire est dévalorisée "it is commonplace to hear politicians reassure us that they are serious about beating inflation. These statements are correctly regarded with skepticism » (Backus, Driffil, 1985, p. 536). Les deux auteurs concluent que l'on devrait favoriser la mise en place de banquiers centraux autonomes vis-à-vis du pouvoir politique, de manière à rendre plus crédibles les politiques anti-inflationnistes ${ }^{11}$. Ainsi, les propositions théoriques des années 1980 mettant en avant les anticipations des agents et une lecture des politiques en termes de public choice auront des implications sur le type de mandat à accorder aux banquiers centraux. De ce point de vue, il existe une cohérence totale entre l'approche de Kydland-Prescott puis Barro-Gordon et Backus-Drifill.

11 Par la suite, la littérature théorique analysant la communication des Banques Centrales fut reprise au sein du courant de la Nouvelle Ecole Keynésienne, focalisée également sur la formation des anticipations des marchés à propos de la politique monétaire.

12 Ainsi, la notion de crédibilité va s'enrichir de celle de réputation. Cette idée va être développée par les apports des auteurs néo-keynésiens (voir Clarida, Galí, Gertler, 1999 et les articles cités plus avant) centrés sur la remise en cause de l'hypothèse d'information parfaite. Une part importante de la crédibilité est donc construite sur le discours qui doit permettre aux agents de guider leurs anticipations par rapport aux variations de l'environnement économique dans la mesure où l'information des agents privés est imparfaite alors que celle dont dispose la Banque centrale est supposée plus complète. Pour éviter que cette asymétrie d'information n'entraîne de forte déstabilisation des anticipations, le discours de la Banque centrale doit expliciter les objectifs qu'elle se fixe, les moyens pour y parvenir ainsi que donner des explications sur l'évolution de l'environnement macroéconomique qui justifierait à ses yeux une évolution de la politique monétaire. La Banque centrale, dont l'activité se situe au centre d'intérêts contradictoires, doit donc délivrer un discours millimétré. Par conséquent, une politique de communication efficace améliorerait le degré de connaissances du public en réduisant l'asymétrie d'information entre celui-ci et l'institut d'émission qui produit des informations "supérieures", significatives ${ }^{12}$ (plus précises) sur les anticipations d'inflation et les perspectives économiques du secteur privé (Eijfinger, Geraats, 2006).

Dans ces conditions, les banquiers centraux prennent progressivement conscience de l'importance du choix de leur vocabulaire (ce que l'on peut qualifier de «wording»). Cet aspect a d'ailleurs été souligné par Dow et Saville (1988) qui considèrent que lorsque la Banque centrale " parle », cela envoie des signaux qui doivent être précis et clairs.

Woodford $(2003,2005)$ confirme cette tendance en soulignant qu'une politique monétaire efficace ne devait pas seulement contrôler les taux au jour le jour mais affecter également l'évolution des anticipations du marché, en particulier en ce qui concerne l'inflation future. La communication et l'engagement sur la politique monétaire à venir constituent donc un levier primordial de la politique monétaire ${ }^{13}$. La Banque Centrale doit désormais s'engager dans des stratégies de forward guidance (où elle annonce une stabilité de ses taux sur longue période) sur des périodes longues.

Un « consensus » sur les objectifs de la politique économique monétaire s'est donc établi autour du modèle néokeynésien de Clarida, Gali et Gelter (1999). La Banque centrale minimise une fonction de perte où l'on trouve l'inflation et l'écart au P.I.B. potentiel. Les 
objectifs de la Banque centrale consistent à assurer la stabilisation de l'inflation et de la production. La politique budgétaire est jugée inefficace, voire déstabilisatrice, seule la politique monétaire doit intervenir afin de transférer aux agents une information qu'ils ne possèdent pas. À cet effet, la Banque centrale produit des évaluations macroéconomiques, des prévisions et des analyses qui doivent réduire au minimum l'asymétrie d'information des acteurs privés et ainsi assurer une stabilisation des anticipations conduisant à une faible volatilité macroéconomique. Les erreurs de prévision des acteurs doivent être minimales de façon à se situer toujours au plus près du plein emploi des facteurs. La production d'information, leur mise en forme à travers les bulletins mensuels, d'autres supports et les discours des banquiers centraux constituent finalement le second instrument de la politique monétaire ce qui justifie de s'intéresser à la dimension performative du discours de la Banque centrale.

Ainsi, au cours des années 1980 et 1990, le rôle de la communication des Banques Centrales en tant qu'instrument majeur pour guider les anticipations a fait l'objet d'un consensus parmi de nombreux théoriciens. À ceci près qu'au sein de la NEC, le jeu stratégique confrontait le public et les autorités, alors que la NEK associait ces deux entités. Dans les deux cas, on considérait qu'une communication accrue améliorerait l'efficacité de la politique monétaire, de sorte que l'émission d'avis passait du statut de nuisance à celui d'outil-clé.

\section{Comment les banquiers centraux influenceraient l'environnement} économique :

«Annonces » de la Banque centrale

$\rightarrow$ décryptage (médias, académiques, investisseurs)

$\rightarrow$ révision des anticipations de l'ensemble des agents

$\rightarrow$ modifications des comportements (consommation, investissement...)

\subsection{Les canaux de transmission de la communication des Banques centrales}

L'idée que les discours possédaient le pouvoir de transformer la réalité a été disséminée ; en conséquence, les Banques Centrales ont substitué les opérations de «bouche ouverte » (open mouth) à celles d'open market (Guthrie, Wright, 2000). Parallèlement à ces travaux théoriques, on observe une mutation dans les pratiques des Banques Centrales au cours de la décennie 1990, notamment de la part de la FED. Dès février 1994, le Federal Open Market Comitee annonce ses décisions quant à la cible concernant les taux directeurs.

18 À partir des arguments théoriques évoqués précédemment en faveur de la communication des Banques Centrales, on peut identifier les cinq canaux de transmission suivants :

- Tout d'abord, la communication améliore la crédibilité de la Banque centrale concernant son engagement à satisfaire les objectifs (Issing, 1999 ; Woodford, 2001 ; Mishkin, 2004 ou encore Ehrmann et Fratzscher, 2011).

- Par ailleurs, la communication de cet organisme permettrait de guider, d'orienter les anticipations des marchés financiers (Woodford, 2003, Bernanke, 2004, Trichet, 2005 ${ }^{14}$ ), en créant des "nouvelles» (news, par exemple, en faisant évoluer les taux courts dans la direction désirée) et en réduisant les «bruits» (noise, par exemple, en diminuant 
l'incertitude sur les marchés), ce qui orienterait ainsi les prix des actifs. Par conséquent, la communication des Banques centrales améliorerait la cohérence, la transparence et la prédictibilité de la politique monétaire. En outre, cela réduirait également la volatilité et l'incertitude des marchés financiers, pour, au final, développer le bien-être économique ${ }^{15}$. L'institut d'émission est censé produire des informations "supérieures", plus précises sur les anticipations d'inflation et les perspectives économiques du secteur privé (Eijfinger et Geraats, 2006).

- En outre, dans une situation de taux d'intérêt proches de zéro (ou nuls), la communication de la Banque centrale sur les taux futurs anticipés devient «l'essence » de la politique monétaire (Eggertsson et Woodford, 2003 ; Bernanke, Reinhart et Sack, 2004). Le canal majeur porte sur l'effet des changements des taux courts sur les anticipations des taux courts futurs (Blinder, 1998), leur impact sur les taux longs et, par conséquent, sur la demande ${ }^{16}$. La communication permettrait d'impacter les prix des actifs et les taux d'intérêt à plus longue échéance, qui agissent sur les décisions des investisseurs, des entreprises et des États (Bernanke, 2004).

- Enfin, à travers la communication, la Banque Centrale cible son discours en direction des investisseurs qui sont très sensibles à la communication directe de l'institution, puisque sa politique monétaire doit avoir un impact sur les marchés des actions (Fair, 2002). Sa communication peut, par conséquent, exercer des effets à travers ce qui a été labellisé dans la littérature (Kaminsky et Lewis, 1996) sous le terme de « canal de signalement ».

Avant de présenter une analyse textuelle succincte, nous présentons les pratiques concrètes de communication de la $\mathrm{BCE}$, en nous focalisant sur les cibles et les instruments de communication utilisés ainsi que sur le vocabulaire spécifique employé par les présidents successifs, J.-C. Trichet puis M. Draghi.

\section{Les mots de la Banque centrale européenne}

En 1999, la B.C.E. se voit instituée comme « le lieu de la souveraineté monétaire » au sein du Système Européen des Banques Centrales (Creel et Fayolle, 2002, p. 181). Dans le domaine pratique, les principaux objectifs des deux décennies suivantes s'articuleront autour de de la crédibilité et de la transparence ${ }^{17}$. La définition des statuts de la BCE est étroitement liée à la doctrine nouvelle classique reprise par les apports des auteurs néokeynésiens. La Banque centrale n'intervient plus pour régler le cycle, mais pour stabiliser les anticipations et réduire l'asymétrie d'information. Une institution « jeune » comme la BCE devra adopter une conduite de type « faucon » (adverse à l'inflation) afin d'assurer sa crédibilité, qui se construit de manière permanente (Ehrmann et Fratzscher, 2011). Il convient dès lors de déterminer des règles claires, de manière à ce que tout écart puisse être identifié immédiatement par les agents. Dans la zone euro, «l'objectif principal du SEBC est la stabilité des prix. » (article 105 du traité de Maastricht), ce «mandat clair et étroit » permettrait une efficacité maximale (Jabko, 2001). En conséquence, un discours transparent devra accompagner et expliciter les actes de la politique monétaire ${ }^{18}$.

Implicitement ces objectifs contiennent la stabilité financière, nouveau domaine sur lequel les Banques centrales communiquent régulièrement (que ce soit via la publication de rapports de stabilité financière, des discours ou des interviews des membres du comité directeur). S'agissant de la BCE (2011), l'objectif du rapport de stabilité financière (qui fournit un aperçu des sources de risques et de vulnérabilité) vise à promouvoir la prise de conscience dans l'industrie financière et au sein du public du rôle que joue l'institution 
dans la prévention des crises financières et la sauvegarde de la stabilité dans la zone euro: un "canal de coordination" se met en place, réduisant l'hétérogénéité des anticipations et de l'information et conduisant les prix des actifs à refléter de façon plus proche les fondamentaux sous-jacent. Ce canal peut impliquer que la communication pourrait modifier les dynamiques sur les marchés financiers et ce nouvel objectif rend l'institution très soucieuse des réactions des acteurs financiers. Rappelons que dans la théorie standard, les marchés financiers sont censés être efficients -donc autorégulateurs-. La valeur fondamentale des titres s'impose aux opérateurs, en fonction de toute l'information disponible (Fama). Or, dans la pratique les autorités monétaires tiennent de plus en plus compte des représentations et croyances des détenteurs de titres : les Banques Centrales estiment donc que parmi la masse d'informations, l'émission de leurs messages peut influencer les décisions des investisseurs, point non abordé par Fama.

\subsection{La communication de la BCE}

En premier lieu, la communication de la Banque Centrale sur le plan pratique est à destination de diverses audiences telles que les participants aux marchés financiers, le public $^{19}$, les pouvoirs politiques, les « académiques » et des médias.

Différents outils de communication sont ainsi à disposition de la BCE. On peut répertorier :

- le bulletin mensuel de la BCE ;

- sa conférence de presse mensuelle (et sa session de questions-réponses avec la presse), afin de clarifier certaines ambiguïtés ;

- les minutes des réunions du directoire, publiées depuis février 2015 et qui donnent un compte rendu des réunions du Conseil des gouverneurs et des débats qui ont lieu. Cela augmente ainsi la transparence de la BCE, même si le mystère perdure quant aux indications nominatives des votes;

- le témoignage du comité sur les affaires monétaires de l'Europe parlementaire (quatre fois par an) ;

- les discours par le président et/ou les membres du conseil des gouverneurs ainsi que leurs interviews.

Toutefois, dans son bulletin mensuel de novembre 2002 (p. 64), la BCE précise que la conférence de presse mensuelle, tenue par le président et le vice-président, et le bulletin mensuel sont deux des plus importants canaux de communication adoptés. Ils fournissent un résumé global de la politique de l'évaluation pertinente des indicateurs économiques. Il faut, en outre, signaler que l'éditorial du Bulletin Mensuel de la BCE et la déclaration introductive de la conférence de presse rapportent la même information concernant l'orientation de sa politique. Ainsi, l'éditorial est rendu disponible au public en étant publié une semaine après chaque réunion de politique monétaire.

Étant donnée la médiatisation de la conférence de presse et du bulletin mensuel de la BCE, il n'est pas étonnant que la littérature empirique ${ }^{20}$ se soit focalisée sur l'analyse du contenu de ces deux moyens de diffusion.

Par ailleurs, on peut observer une certaine évolution dans le temps des pratiques de communication de la BCE qui se traduit par une amélioration quantitative et qualitative vers plus de clarté et de transparence. En effet, d'après l'étude de Galardo et Guerrieri (2015) entre 2008 et 2014, la part consacrée à l'explication des décisions de politique 
monétaire et aux risques sur la stabilité financière, a plus que doublé durant cette période et représente $40 \%$ du temps de la conférence de presse, contre moins de $10 \%$ en 2002 . Cela traduit, par conséquent, un certain apprentissage de la communication auprès des marchés, de la part de la BCE.

En outre, la communication dépend de l'émetteur (un membre individuel ou le directoire entier), ce qui peut poser des problèmes de « cacophonie » en cas de divergence entre les membres $^{21}$.

On a récemment a accordé beaucoup d'attention au vocabulaire employé (adjectifs, adverbes, emploi du futur) par les présidents de l'institution. On peut ainsi relever que Trichet (2003-2011) utilisait certains mots codés (De Haan et Jansen, 2009, p. 11) : le mot " vigilance » traduisait un avertissement aux marchés sur une hausse prochaine des taux, l'adjectif « équilibrées » était relié aux anticipations d'inflation sur la stabilité des prix ou la croissance... Notons qu'après 2008, que le terme « risque » fut beaucoup plus utilisé, particulièrement durant la crise de la zone euro en 2011 (Légé et Marques Pereira, 2013). Draghi, quant à lui, utilise les adjectifs « modéré » concernant l'économie et l'inflation, ou bien « accommodant » pour la politique monétaire de soutien de la BCE (Carré, 2014). Il emploie également des déclarations chocs, dont le fameux «What ever it takes » du 26 juillet 2012 (voir plus bas). Enfin, il évoque le futur à travers l'utilisation des mots « forward guidance » ou " éléments prospectifs » et n'hésite pas à utiliser des expressions claires telles que « les taux d'intérêt vont rester bas sur une période prolongée ».

Comparaison du contenu lexical de la communication de Trichet et de Draghi

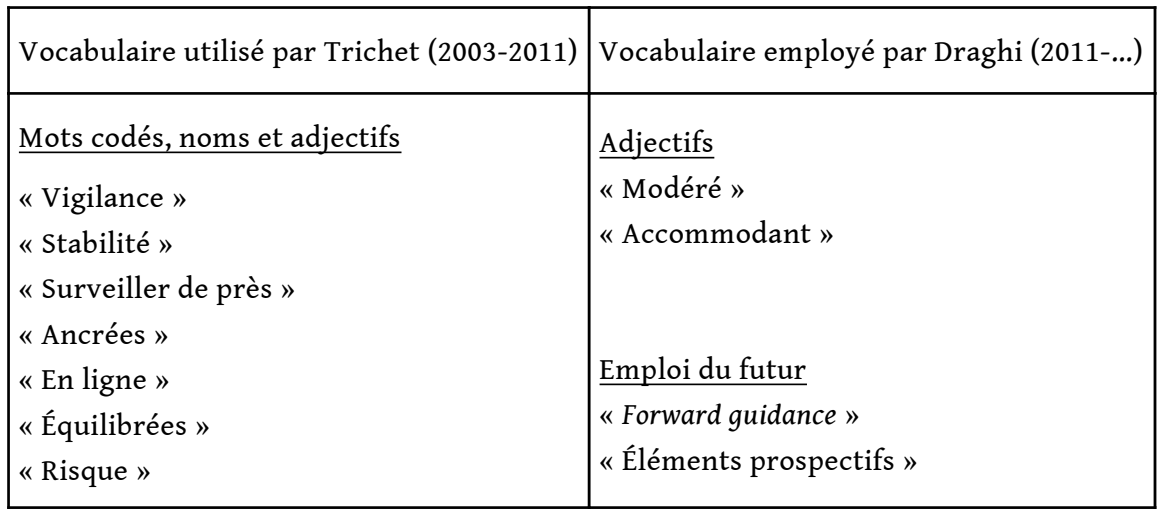

\subsection{Un indicateur de tonalité du discours}

Dans ce travail, nous mesurerons la tonalité du discours de la BCE en utilisant la méthode de traitement textuel du discours développé par Sadique, In, Veeraraghavan, et Wachtel (2013) sur le Beige Book utilisé par le Fed (sur les conditions économiques aux États-Unis). Ces économistes-financiers caractérisent la tonalité du rapport puis étudient son contenu prédictif en utilisant un logiciel d'analyse textuelle. Ils cherchent à déterminer si le Beige Book donne des indications sur les points de retournement de l'activité économique, et s'il existe des liens avec les variables financières (taux d'intérêt, rendement des actions, volatilité et volume des transactions). Ces auteurs montrent que le ton général du Beige Book est en phase avec le cycle de la production industrielle, du chômage et de la situation économique générale (durant les périodes de croissance, la tonalité positive l'emporte et inversement). En revanche, cette tonalité qui pourrait affecter la volatilité du marché des 
actions et le volume des transactions, n'influence pas les rendements. La méthode utilisée par Sadique et al. permet un examen automatique des éditoriaux qui ne requiert pas d'interprétations qualitatives du discours, comme c'était le cas dans de précédentes études sur le Beige Book (Armesto et al., 2009 ; Zadovny et Ginther, 2005).

Dans notre travail, nous recensons tout d'abord le nombre de mots de chaque éditorial du Bulletin mensuel de la B.C.E. (sur la période comprise entre février 1999 et septembre 2014), extraits grâce au logiciel R et au package TM (Text Mining) qui permet de compter les mots en évacuant la ponctuation et les chiffres. Puis, selon les connotations positives (exemple : «croissance ») ou négatives (exemple : « récession») établies par le lexique proposé par le dictionnaire Harvard $\mathrm{IV}^{22}$, on construit des indicateurs de tonalité positive (optimiste), négative (pessimiste), mais également synthétique en faisant la soustraction entre les deux. En outre, deux autres indicateurs sont créés, pour ce qui est de la variation de la tonalité, à savoir "amélioration» et "dégradation", décrivant ainsi des augmentations ou des diminutions du sentiment à partir d'un indicateur synthétique de la variation du ton de la Banque centrale.

31 Ainsi, les mots, en fonction de leur catégorie (positif, négatif, amélioration ou détérioration de ton) sont additionnés par bulletin mensuel et exprimés en pourcentage puisqu'ils sont rapportés à l'ensemble des mots du Bulletin mensuel.

On obtient au final les séries temporelles mensuelles suivantes (graphiques 1 et 2, cidessous).

Graphique 1. Ton des éditos en pourcentage de l'ensemble des mots

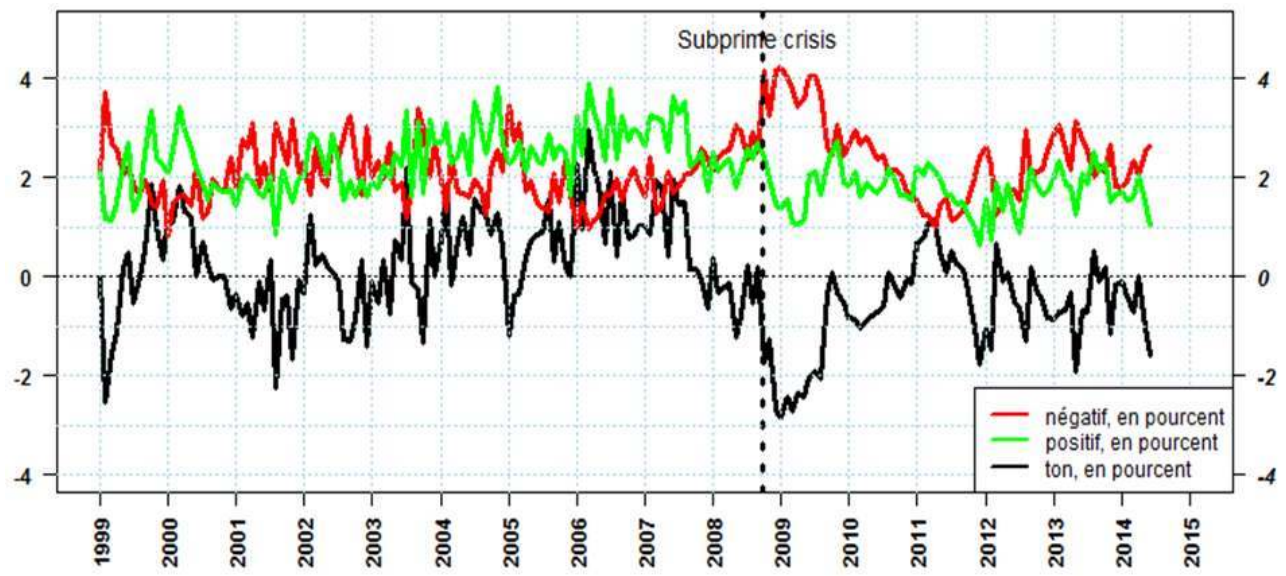

Sur ce graphique, on observe, au mois d'octobre 2008, une augmentation sensible des occurrences de mots à tonalité négative, qui représentent habituellement autour de $2 \%$ de l'ensemble des mots. Entre la fin 2008 et le début 2009, leur fréquence va dépasser $4 \%$ sur trois mois. La fréquence de mots négatifs est doublée par rapport à une situation normale, ce qui permet de valider la pertinence du comptage de mots pour détecter une situation de crise. L'écart entre la fréquence des mots positifs et négatifs, qui représente la variable de ton, est centré autour de zéro, et détecte également correctement la crise des subprime. On observe logiquement une symétrie entre les mots à tonalité négative et positive. Par ailleurs, lorsqu'on observe un pic du ton à $3 \%$ en 2006, cela correspond bien à un croisement des courbes positives et négatives. Nos variables apparaissent relativement pertinentes. Enfin, on peut apercevoir, à la naissance de l'euro, un ton assez 
négatif; les autres points qui s'approchent de $-2 \%$ concernent le 11 septembre 2001, la crise de la zone euro fin 2011 et sa résurgence début 2013, lors de la crise chypriote.

Graphique 2. Variation de ton des éditos en pourcentage de l'ensemble des mots

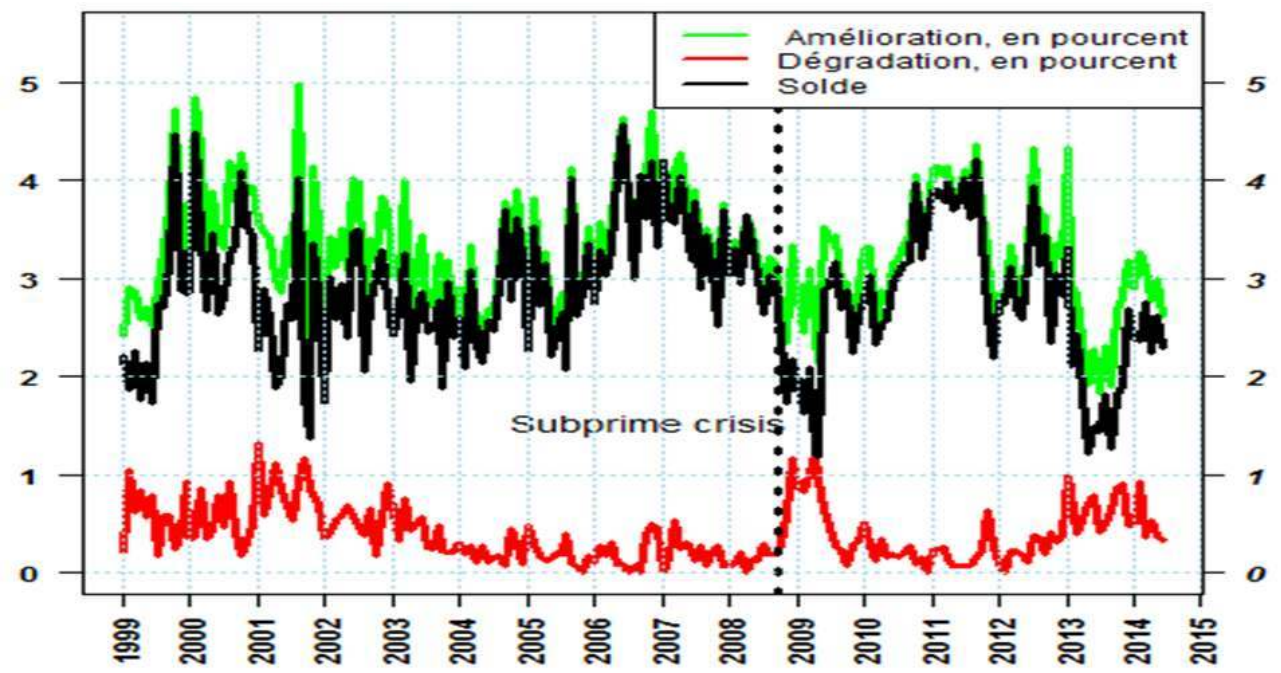

Les indicateurs d'amélioration, de dégradation et de variation de ton (graphique 2) décrivent également bien les événements extrêmes, mais semblent moins pertinents que les variables de tons, dans la mesure où les mouvements d'ensemble de la variable d'amélioration et de dégradation paraissent assez proches. Ainsi, l'information apparaît plus bruitée et le signal moins précis. La faible variance de l'indicateur de changement (qui représente l'écart entre les deux variables précédentes) pourrait être liée au nombre de mots retenu pour chaque tonalité, mais également à l'ambivalence des termes lorsqu'on les analyse un à un. Autrement dit, fait-on face à une augmentation de l'activité ou bien à une augmentation du chômage? Dans les deux cas, l'analyse quantitative du comptage de mots retiendra une tonalité positive, alors que la phrase pourra, en fait, décrire un phénomène négatif. Il s'agit d'une des limites de l'exercice par comptage de mots. Certes, cette technique permet de sortir de toute analyse qualitative du discours et d'éviter les biais d'interprétation, mais de fait, on perd une partie de l'information. Des techniques linguistiques plus avancées, à partir de l'analyse granulaire des corpus ${ }^{23}$, devraient permettre de poursuivre un comptage automatique, non plus des mots, mais des expressions à tonalité positive ou négative, en fonction d'un nombre de mots défini. Toutefois, cela conduit immédiatement à une complexification du traitement de l'information. La technique la plus simple d'un comptage individuel des mots reste donc pertinente.

Notons que cette technique peut générer un biais dans la représentativité des tonalités car, dans le dictionnaire retenu, les mots correspondant au ton négatif sont beaucoup plus nombreux, avec 2349 occurrences contre seulement 354 occurrences pour les mots positifs. Les expressions représentant des variations de ton sont également beaucoup moins nombreuses (98 occurrence d'amélioration de ton et 79 pour identifier une dégradation de la tonalité). Ces écarts importants du nombre d'occurrences permettent sans doute de comprendre l'impression de bruit ressenti à lecture du second graphique. Cela peut également s'expliquer par le fait que, malgré un faible nombre de termes, la fréquence d'apparition des termes indiquant une variation de ton est plus forte que celle des mots décrivant une tonalité (qui sont toutefois plus nombreux). Cette situation laisse 
à penser que le recours aux mots de variation de tonalité est moins discriminant en fréquence absolue. En revanche, leur différence à travers la variable de changement permet de bien identifier les périodes de crise, même si cette variable présente une variance moins forte que la variable de ton. Finalement, les variables de tonalité paraissent individuellement plus informatives, sans doute en raison du nombre de leur occurrence. En revanche, au niveau de l'indicateur représentant l'écart de ton, c'est l'indicateur de variation de ton qui semble le plus efficace pour indiquer un changement de tonalité.

Ces observations tirées de la lecture des graphiques 1 et 2 montrent qu'il y a bien une "mise en mots» du monde économique par le Banquier central. Encore faut-il déterminer vers quel type d'acteurs cet exercice est destiné.

\section{L'audience visée par les discours}

37 La littérature économique des années 1980-90 a mis en avant la notion de crédibilité sensée conforter la parole des autorités publiques. Une crédibilité forte aurait d'autant plus de chances de modifier le comportement des acteurs. Les relations qu'entretiennent les échanges monétaires avec la confiance ont été soulignées à de multiples reprises (pour une synthèse, voir Aglietta, Orléan, 1998). Etymologiquement, crédit et confiance partagent la même racine latine. En revanche, l'attribution d'une dimension rituelle à la parole des banquiers centraux, "grands prêtres" de la monnaie, est plus récente. ${ }^{24}$ De fait, un dispositif institutionnel s'est mis en place autour de leurs prises de parole, en particulier en Europe. Face aux soupçons de dé-légitimation provoqués par l'indépendance des banquiers centraux -particulièrement sensibles dans le cas de la BCE qui apparaît comme « déterritorialisée » (Aglietta, 2011, p. 11)-, il convenait de d'attribuer aux messages émis un poids symbolique. Mais pour exercer des effets significatifs, un discours doit respecter un certain nombre de conditions.

\subsection{La valeur fiduciaire du porte-parole}

Austin prévenait : "Nous pouvons certes obtenir certaines suites d'actes perlocutoires par des moyens non conventionnels (" pas très réguliers » comme on dit) » (Austin, 1962, p. 126). Prenons un exemple de prise de parole «non conventionnelle» qui peut aider à préciser certaines notions : ce discours a été proféré hors bulletin mensuel de la B.C.E lors d'une conférence de presse le 26 juillet 2012. Avant même d'évoquer le « whatever it takes $»^{25}$, le président du directoire de la B.C.E. commence par l'évocation d'un «mystère de la nature »: "The euro is like a bumblebee. This is a mystery of nature because it shouldn't fly but instead it does. » Ainsi, malgré une constitution défaillante, l'euro aurait mystérieusement subsisté. Le président de la B.C.E. n'explique pas les raisons de cette défaillance, mais le miracle s'est bien produit. Ensuite, il avance que l'institution est " prête à tout », c'est-àdire à outrepasser certaines limites ${ }^{26}$. C'est l'aspect le plus communément retenu. Toutefois, Draghi ajoute un «believe me » qui évoque plus l'acte de foi que l'appréciation objective. Armé de la confiance de ses auditeurs, le «bourdon/euro » pourrait donc reprendre son envol? Rappelons que la prime de risque sur le taux des obligations des pays de la zone euro en difficulté (Espagne, en particulier) à 10 ans s'est bien produite, alors même qu'aucune action concrète ne fut engagée par la BCE. Dans les médias, on commença alors à qualifier Draghi de «magicien» ... Parfois qualifiée de «miracle 
monétaire » dans les médias, cette prise de parole apparaît plutôt comme un «miracle financier ", puisque ce sont les créanciers qui se sont manifestés, pas les utilisateurs de la monnaie Euro ${ }^{27}$.

Sur le versant sociologique, cet acte de langage a été abordé par Holmes «Draghi, in contrast to his predecessor, was carefully disentangling the ECB from the thrall of the Bundesbank. (...) The challenge he faced was to model a communicative relationship by which the public was persuaded to collaborate in the restoration of faith and credit in an irrevocable monetary union. " (Holmes, 2015, p. 12). Selon lui, en voulant restaurer la foi dans une union monétaire irrévocable, le président du directoire de la BCE reprenait le contrôle de la narration. " What Draghi admitted was that the currency had, under pressure from the bond market, become untethered from narrative management of the technocratic officialdom of central banks. He further conceded that if central banks lose control of the monetary story, lose control of their communicative relationship with the public, there are alternative narratives available for stabilizing or destabilizing the link between money and the existential circumstances of the public. »(Holmes, 2014, p. 213).

40 Majoritairement, les études de type sociologiques insistent sur l'impact du discours dans les médias et sur le public: "the public broadly must be recruited to collaborate with central banks in achieving the ends of monetary policy, namely 'stable prices and confidence in the currency'." (Holmes, 2015, p. 2.). Lorsqu'il évoque l'impact des mots, Holmes reprend les outils économiques standard qui transforment les agents économiques en un "public » aux contours flous (mais aux anticipations extrêmement fines). Les agents sont sensés réagir, notamment lorsque le niveau de l'inflation risque d'être modifié, mais ils ne sont jamais caractérisés précisément (sphère publique/privée, créanciers/débiteurs...). Selon les présupposés de la théorie standard, on postule que ce public est à même d'interpréter correctement le vocabulaire utilisé.

41 À la suite de Butler, les sociologues commencent à étudier la théâtralité de certaines situations : dans les cas des banquiers centraux, cela amène à étudier l'effet de certaines tournures de phrase lors des rencontres avec la presse (dernier exemple, Velthuis, 2015). Dans ce cas, le "public» visé se restreint souvent aux journalistes présents lors de l'intervention. Mais quelle est l'audience visée en dernier ressort?

En premier lieu, l'audience est constituée d'initiés car il existe un «caractère relativement ésotérique (en apparence tout au moins) des questions monétaires, entretenu par la formation de groupes d'interprètes savants qui maintient les profanes à bonne distance" (Lebaron, 2006, p. 19). Dans ce type d'opération où l'acte de foi est déterminant, le moment de l'allocution importe beaucoup : «L'efficacité magique de ces actes d'institution est inséparable de l'existence d'une institution définissant les conditions (en matière d'agent, de lieu ou de moment...) qui doivent être remplies pour que la magie des mots puisse opérer.» (Bourdieu, 2001, p. 108). Les liens entre le locuteur et son audience commencent à être précisés. Selon Bourdieu, toujours, « le porte-parole autorisé ne peut agir par les mots sur d'autres agents (...) que parce que sa parole concentre le capital symbolique accumulé par le groupe qui l'a mandaté et dont il est le fondé de pouvoir » (Bourdieu, 2001, p. 163). Si l'on suit cette analyse, le banquier central apparaît comme le porte-parole des détenteurs de créances. Dans cette configuration, la communication de la Banque Centrale sert à actualiser régulièrement le contrat qui la lie aux acteurs financiers, car les créditeurs souhaitent être rassurés régulièrement. Bourdieu évoque la « valeur fiduciaire» du locuteur/mandataire (idem, p. 241). Ainsi se constitue un jeu de miroirs entre la Banque centrale et les marchés financiers, au sein d'un cadre 
institutionnel qui permet au discours de devenir crédible et former des prévisions autoréalisatrices.

\subsection{Perlocution et conventions sur les marchés financiers}

Le poids accordé aux énoncés de certains locuteurs est lié au caractère très incertain des prévisions financières ("The weight of stories in financial markets can be understood as a response to the high level of uncertainty prevailing in these markets. ", Beckert, 2013, p. 28). Dans ce contexte, le message émis par le banquier central fournit un point focal qui servira de référence aux investisseurs et réduira l'incertitude ("Together with open market operations, the economic narratives of central banks thus become the second main determinant for price developments. Put differently, uncertainty is being reduced by discursive practices that rely on strategic rhetorical action », Nelson et Katzenstein 2010, p. 32).

En pragmatique du langage, si l'effet produit par un énoncé conduit à modifier les représentations des interlocuteurs, il sera qualifié de perlocutoire. Dans ce cas, le discours entraine une modification du comportement des auditeurs -ou « consommateurs » (Bourdieu)- du discours. En provoquant une révision des anticipations (et des évaluations financières), l'effet de la prise de parole joue sur les représentations des agents. Cette notion de perlocution peut finalement être reliée à l'approche conventionnaliste de la formation des anticipations, et plus largement de coordination des agents en univers incertain (Orléan, 1999), à savoir que le consensus des acteurs contribuerait à transformer leur vision de l'avenir. Cette révision les amènerait à revoir leurs évaluations financières. Orléan précise ce que peut être un marché financier guidé par l'auto-référentialité : «Les intervenants y sont perpétuellement à la recherche des saillances aptes à recueillir l'agrément du marché. Face à une nouvelle information, c'est à cette activité que sont centralement affectées les capacités cognitives des investisseurs : chacun mesure la capacité de telle ou telle information à répondre aux attentes conventionnelles du marché telles qu'elles s'expriment à un moment donné. » (Orléan, 2004, p. 266) ${ }^{28}$

Ainsi, la parole du banquier central modifie de facto l'environnement informationnel des agents, mais ne transforme pas le réel: la Banque centrale cherche à influencer les anticipations en produisant un cadre institutionnel, celui de sa politique de communication. Il y a donc autoréférentialité, le but ultime de la politique monétaire étant de préserver la valeur des créances, plutôt que de servir la relance. Ainsi, la possibilité pour les banques centrales de contrôler l'ancrage de l'inflation nécessite un état des rapports de force socio-politique spécifique. Le dispositif qui rend perlocutoire la parole du banquier central est une alliance de propositions théoriques et de rapports économiques. Dans un premier temps, la mise en avant des anticipations dans la théorie a contribué à transformer l'exercice de la politique monétaire en un gouvernement des anticipations. À propos de la théorie des anticipations rationnelles, Aglietta et Orléan notaient qu'elles dotent «les sujets privés d'une omniscience vis-à-vis du système économique dans lequel ils vivent. (...) Les marchés privés se chargent de tout. L'action des autorités ne peut déterminer que les valeurs nominales. " (Aglietta, Orléan, 1984, p. 228).

Boucle perlocutoire entre Banque Centrale et marchés

Banquier central ayant acquis une valeur fiduciaire auprès des détenteurs de créances 
$\rightarrow$ « annonce » en direction de ces créanciers

$\rightarrow$ anticipations confortées ou révisées

$\rightarrow$ ajustement sur les marchés de créances

$\rightarrow$ attente d'une nouvelle annonce du fondé de pouvoir...

On retrouve ainsi des aspects importants des sciences de la communication: la communication vise souvent à faire l'économie d'actes réels. Ses actes langagiers permettent de calmer les attentes en laissant entendre que tout ce qu'elle doit entreprendre est fait (mais la communication ne saurait à elle seul remplacer des politiques de redistribution et de relance budgétaire nécessaire à reprise robuste économique et socialement). La littérature sur l'efficacité du discours permet de légitimer l'état du rapport de force actuel où la politique monétaire se trouve captée par les intérêts privés car les "marchés » pourraient sanctionner une politique monétaire jugée insuffisamment accommodante. Au final, le virage communicationnel des banques centrales constitue un avatar de la financiarisation de l'économie.

\section{Conclusion}

L'accent mis sur la communication des Banques Centrales était, on l'a vu, cohérent avec l'argumentaire défendant la transparence et l'indépendance des instituts d'émission. Dans la lignée des propositions potentiellement performatives de Kydland et Prescott, il convenait de confier la conduite de la politique monétaire à des responsables dénués de préoccupations électoralistes. Ceci aurait pour but de lester leur discours d'un poids conséquent, les politiques ne respectant pas suffisamment leur propre parole. Cela s'est concrétisé à travers la construction de la Banque Centrale Européenne. Cette indépendance avait pour objectif de régler le dilemme des politiques économiques : un pouvoir politique serait toujours tenté de recourir à la politique monétaire pour réduire le chômage au risque de conduire à une inflation plus importante et finalement réduire la croissance potentielle. Seule l'indépendance vis-à-vis des pouvoirs élus autoriserait une politique monétaire crédible. Ainsi, une rhétorique d'expert soustraite aux préoccupations électoralistes permettrait-elle d'ancrer solidement les anticipations et d'agir sur certaines variables macro-économiques. Ainsi, l'attention aux mots utilisés par les banquiers centraux est devenue très importante, et leurs inflexions lexicales sont désormais décryptées avec soin.

Pour reprendre la question posée de manière provocante par la note de Natixis (2014), on peut se demander si la montée en puissance du thème de la communication des Banques Centrales ne se résume pas à « beaucoup de bruit pour rien »? En effet, « Les décisions, les annonces de la $\mathrm{BCE}$ ont donc en réalité très peu de conséquences pratiques sur l'économie réelle.» (Flash Natixis, 2014, p. 1) Aurait-on surestimé la fonction performative du discours des banquiers centraux? Si oui, pour quelles raisons? D'un point de vue sociologique, on peut considérer que «l'espace occupé par les banquiers centraux constitue un sous-espace du champ globalisé du pouvoir » (Lebaron, 2008, p. 126). Dès lors, la hausse du volume de discours émis correspond à une volonté d'ancrage social. Face aux soupçons de dé-légitimation provoqués par l'indépendance des banquiers centraux -particulièrement sensibles dans le cas de la BCE qui apparaît comme «déterritorialisée ${ }^{29}$-, il convenait de d'attribuer aux messages émis un poids symbolique. Ainsi a été disséminée l'idée que les discours émis étaient performatifs. D’où 
l'écho souvent exagéré qu'ils provoquent ${ }^{30}$. Rappelons d'ailleurs que Milton Friedman était opposé à l'indépendance, qui aurait pour effet d'empêcher le contrôle démocratique des politiques monétaires ${ }^{31}$ et de rendre ces politiques «terriblement tributaire(s) des personnes » (Friedman, 1962, p. 213).

En définitive, le croisement des approches économiques et sociologiques peut s'avérer fertile pour étudier la politique de communication des Banques Centrales, à condition de préciser les contours du " public » visé par les discours. Si les mots ont une influence, il convient de se poser la question du pouvoir. Suivant l'argumentaire de Bourdieu, on propose d'appréhender l'émetteur du discours autorisé comme un «mandataire légitime " (Bourdieu, 2001, p. 112). Le banquier central apparaît comme le porte-parole des détenteurs de créances, ses actes de langage sont calibrés dans ce but, et les approches en terme de performativité gagneraient à étudier cette voie de recherche.

\section{BIBLIOGRAPHIE}

Aglietta, M. (2011), « Principaux enseignements du rapport », dans Finances publiques : l'épreuve de vérité pour la zone euro, Institut CDC pour la Recherche, Paris, pp. 4-15.

Aglietta, M., Orléan, A. (1984), La violence de la monnaie, PUF, Paris.

Aglietta, M., Orléan, A. (1999) sld, La Monnaie souveraine, O. Jacob, Paris.

Aitken R. (2007), Performing Capital: Towards a Cultural Economy of Popular and Global Finance, Palgrave Macmillan, Basingstoke.

Armesto, M. T., Hernández-Murillo, R., Owyang, M., Piger, J. (2008), « Measuring the information content of the Beige Book: a mixed data sampling approach », Working paper series, Federal Reserve Bank of St. Louis, Research Division.

Austin, J. (1962), How to Do Things with Words, Oxford University Press/ Quand dire, c'est faire, Seuil, Paris, 1970.

Backus, D., Driffill, J. (1985), « Inflation and Reputation », The American Economic Review, 75 (3), pp. 530-538.

Barro, R., Gordon, D. (1983), « Rules, Discretion, and Reputation in a Model of Monetary Policy », Journal of Monetary Economics, 12, pp. 101-21.

Banque Centrale Européenne (2002), Bulletin Mensuel de la BCE, novembre 2002.

Beckert, J., 2013, « Imagined futures: fictional expectations in the economy », Theory and Society, Volume 42, 3, pp. 219-240

Bennani, H., Farvaque, E. (2014), « Speaking in tongues? Diagnosing the consistency of central banks' official communication », Working Paper.

Berger, H., De Haan, J. et Sturm, J-E. (2011), « Does money matter in the ECB Strategy? New evidence based on ECB communication ", International Journal of Finance and Economics, pp. 16, $16-31$. 
Bernanke, B. (2004), « Gradualism, Speech 540, Board of Governors of the Federal Reserve System. Bernanke, B., Reinhart, B. et B.P. Sack, (2004), « Monetary Policy Alternatives at the Zero Bound: An Empirical Assessment », Brookings Papers on Economic Activity, 2, pp. 1-100.

Bibow J. (2010), « A Post Keynesian Perspective on the Rise of Central Bank Independence: A Dubious Success Story in Monetary Economics », Levy Economics Institute, working paper 625.

Blinder, A. (2009), « Talking about Monetary Policy: The Virtues (and Vices?) of Central Bank Communication », BIS Working Paper no. 274.

Blinder, A. (2004), The Quiet Revolution, Central Banking goes modern, Yale University Press.

Blinder, A., Ehrmann, M., Fratzscher, M., De Haan, et Jansen, D-J. (2008), « Central Bank communication and monetary policy: a survey of theory and evidence ", Journal of Economic Literature, 46 (4), pp. 910-945.

Bourdieu, P. (1996), « Contre la 'pensée Tietmeyer', un welfare state européen », Libération, 25 octobre.

Bourdieu, P. (2001), Langage et pouvoir symbolique, Fayard, Paris

Brasset J., Clarke C. (2012), « Performing the Sub-Prime Crisis: Trauma and the Financial Event », International Political Sociology, 6, pp. 4-20.

Brisset, N. (2012), « Deux approches de l'influence du discours économique sur les phénomènes sociaux », Revue de philosophie économique, 2, vol. 13, p. 25-62.

Brisset, N. (2014), « Performer par le dispositif? Un retour critique sur la théorie de la performativité », L'Année sociologique, 1, vol. 64, pp. 217-246

Brunner, K. (1981), « The Art of Central Banking », Center for Research in Government Policy and Business, University of Rochester, Working Paper GPB 81-6.

Butler, J. (1997), Excitable speech: a politics of the performative, Londres, Routledge.

Callon, M. (1998), « The embeddedness of economic markets in economics », in M. Callon, (eds), The Laws of the Markets, Oxford Blackwell, pp. 1-57.

Callon M., Muniesa, M. (2008), « La performativité des sciences économiques », CSI working papers series, 10.

Callon, M. (ed.) (1998), The Laws of the Markets, Blackwell, Oxford

Carré, E., (2005), «Central Bank Communication and the New Consensus », Sciences Po Bordeaux et Université Montesquieu-Bordeaux IV.

Carré, E. et E. Le Héron, (2006), « Credibility versus confidence in monetary policy » in L.R. Wray et Forstater, M., (eds), Money, Financial Instability and Stabilization Policy, Edward Elgar, pp. 58-84.

Carré, E. (2014), « Communication de la BCE et crise financière », communication aux journées

Crise de l'euro, effets discursifs et changements institutionnels, Amiens, 3-5 décembre 2014

Clarida, R., Galí, J., Gertler, M., (1999), « The Science of Monetary Policy: A New Keynesian

Perspective ", Journal of Economic Literature, vol. XXXVII, pp. 1661-1707

Clarke C., Roberts A. (2014), « Mark Carney and the Gendered Political Economy of British Central Banking », British Journal of Politics and International Relations, 2014: 1-23

Couppey-Soubeyran, J., (2012), L'économie Mondiale 2012, La Découverte, pp. 52-67. 
Créel J., Fayolle, J. (2002), « La Banque Central Européenne ou le seigneur des Euros », Revue de l'OFCE, pp. 175-210.

De Haan, J., Jansen, D-J. (2009), « The communication policy of the European Central Bank: an oversiew of the first decade », DNB Working Paper, 212, juin 2009.

De Vroey, M., Malgrange, P., (2007), « Théorie et modélisation macro-économiques, d'hier à aujourd'hui », Revue française d'économie. vol. 21, 3, pp. 3-38

Desmedt, L., Llorca, M. (2012), « The 'Esoteric' Discourse of central bankers in time of crisis », Association of Heterodox Economics conference, Paris, juillet 2012.

Dow, S., Klaes, M. et Montagnoli, A. (2009), « Risk and uncertainty in central bank signals: an analysis of monetary policy committee minutes », Metroeconomica, 60 (4), pp. 584-618.

Dow, S., Saville, I.D. (1988), A Critique of Monetary Policy: Theory and British Experience, Oxford Clarendon Press.

Eggertsson, G., Woodford, M. (2003), « The Zero Bound on Short-Term Interest Rates and Optimal Monetary Policy », Brookings Papers on Economic Activity, 1, pp. 139-211.

Ehrmann, M., Fratzscher, M. (2008), « Purdah - On the Rationale for Central Bank Silence Around Policy Meetings ", Journal of Money, Credit and Banking, 41, pp. 517-528.

Ehrmann, M., Fratzscher, M. (2011), « Politics and monetary policy », The Review of Economics and Statistics, 93(3), pp. 941-960.

Eijffinger, S., Geraats, P. (2006), “How transparent are central banks?”, European Journal of Political Economy, 22(1), pp. 1-21.

Fair, R. (2002), "Events that shook the market”, The Journal of Business, 75(4), pp. 713-732.

Feinerer I. (2008), “An introduction to text mining” in R. R News, 8(2), pp. 19-22.

Feinerer, I., Hornik, K. et Meyer, D. (2008), “Text mining infrastructure”, Journal of Statistical Software, 25(5), pp. 1.

Friedman, M. (1962), « Les solution institutionnelles au problème de la direction monétaire », in Inflation et systèmes monétaires, Calmann-Lévy, Paris, 1969.

Galardo, M., Guerrieri, C., (2015), "More than words: communication as a new monetary policy instrument", Working Paper University Luiss Guido Carli.

Geraats, P.M. (2002), “Central bank transparency”, Economic Journal, 112 (483), pp. 532-565.

Gerlach, S. (2004), "Interest rate setting by the ECB: words and deeds", CEPR Discussion Paper, vol. 4775, Centre for Economic and Policy Research, London.

Gerlach, S. (2007), "Interest rate setting by the ECB, 1999-2006: Words and deeds", International Journal of Central Banking, 3, pp. 1-45.

Guthrie, G., Wright, J. (2000), “Open mouth operations”, Journal of Monetary Economics, 46, 2, pp. 489-516.

Hall, R. B. (2008), Central Banking as Global Governance: Constructing Financial Credibility, Cambridge University Press, Cambridge.

Harris, S., (2008), Ben Bernanke's Fed: The Federal Reserve After Greenspan, Harvard Business Press.

Heinemann, F. et K. Ullrich, (2007), "Does it pay to watch central bankers lips? The information content of ECB wording”, Swiss Journal of Economics, 143(2), pp. 155-185. 
Holmes, D.R. (2009) « Economy of Words », Cultural Anthropology, vol. 24, 3, pp. 381-419.

Holmes, D.R. (2014), Economy of Words: Communicative Imperatives in Central Banks, University of Chicago Press, Chicago.

Holmes, D.R. (2015), « Public currency: Anthropological labor in central Banks », Journal of Cultural Economy: http://dx.doi.org/10.1080/17530350.2015.1068700

Issing, P. (1999), "The monetary policy of the ECB in a world of uncertainty", speech at the conference on "Monetary policy-making under uncertainty", 3-4 December 1999, Francfort.

Issing, O. (2005), "Communication, Transparency, Accountability: Monetary Policy in the TwentyFirst Century", Federal Reserve Bank of St. Louis Review, 87(2), pp. 65-83.

Jabko, N. (2001), « Expertise et politique à l'âge de l'Euro », Revue Française de Science Politique, 51 (6), pp. 903-931.

Jansen, D-J (2011), "Has the clarity of central bank communication affected financial markets? Evidence from Humhrey-Hawkings testimonies", Contemporary Economic Policy, 29 (4), pp. 494-509.

Kaminsky, G., Lewis, K.K. (1996), “Does foreign exchange intervention signal future monetary policy?”, Journal of Monetary Economics, 37 (2-3), pp. 285-312.

Karl, A. (2013), « ‘Bank talk,' performativity and financial markets », Journal of Cultural Economy, 6:1, pp. 63-77

Krippner, G. (2007), « The Making of US Monetary Policy: Central Bank Transparency and the Neoliberal Dillema », Theory and Society, Volume 36, 6, pp. 477-513

Kydland, F., Prescott, E. (1977), "Rules Rather than Discretion: The Inconsistency of Optimal Plans," Journal of Political Economy, 85, pp. 473-93.

Lebaron, F. (2006), Ordre monétaire ou chaos social ? La BCE et la révolution néolibérale, Editions du croquant

Lebaron, F. (2008), « Central bankers in the contemporary global field of power: a 'social space' approach », Sociological Review, 56 (1), pp. 121-144.

Lebaron, F., (2010), La crise de la croyance économique, éditions du croquant, Broissieux.

Légé, P., Marques Pereira, J. (2013), « Les bégaiements de la parole autorisée durant la crise de la zone euro », communication aux journées d'études Discours de la crise et crise du discours, 5-6 décembre 2013, Amiens.

Loughran, T., McDonald, B. (2011), « When is a liability not a liability? Textual analysis, dictionaries, and 10-Ks », Journal of Finance, vol. 66, 1, pp. 35-65

MacKenzie, D., (2007), « Is Economics Performative? Option Theory and the Construction of Derivatives Markets », in MacKenzie, Muniesa, Siu, pp. 54-86

MacKenzie, D., Muniesa, F., Siu, L., (2007) «Introduction », dans Do Economists make Markets? On the Performativity of Economics, Princeton University Press, pp. 1-19

MacKenzie, D., Millo, Y. (2003), « Constructing a market, Performing Theory: The Historical Sociology of a Financial Derivatives Exchange », American Journal of Sociology volume 109, 1, pp. 107-145.

Mishkin, F.S., (2004), “Can Central Bank transparency goo too far?”, NBER Working Papers 10829, National Bureau of Economic Research. 
Nelson, S., Katzenstein, P. J. (2010), Uncertainty and risk and the crisis of 2008, Ithaca, Cornell University.

Natixis (2014), « La BCE : beaucoup de bruit pour rien », Flash 770, octobre 2014.

Orléan, A. (1999), Le pouvoir de la finance, Paris, O. Jacob.

Orléan, A. (2004), « Efficience, finance comportementale et convention : une synthèse théorique ", in Les crises financières, rapport du CAE, pp. 241-270.

Orléan, A. (2011), L'empire de la valeur, Seuil, Paris

R Development Core Team (2005), R: A language and environment for statistical computing. $R$ Foundation for Statistical Computing, Vienna, Austria. ISBN 3-900051-07-0, URL: http://www.Rproject.org.R et package TM.

Rogoff, K. (2014), « Faut-il faire des banquiers centraux les oracles modernes ? », Les Echos, $26 / 11 / 2014$.

Rosa, C., Verga, G. (2005), « Is ECB communication effective? », CEP Discussion Paper, vol. 682. LSE, London.

Rosa, C., Verga, G. (2006), « The impact of Central Bank announcements on asset prices in real time: testing the efficiency of the Euribor futures market », CEP Discussion Papers, 0764, Centre for Economic Performance, LSE.

Rosa, C., Verga, G. (2007), « On the consistency and effectiveness of central bank communication: evidence from the ECB », European Journal of Political Economy, 23, pp. 146-175.

Sadique, S., In, F., Veeraraghavan, M., et P. Wachtel, (2013), « Soft information and economic activity: Evidence from the Beige Book », Journal of Macroeconomics, 37, pp. 81-92.

Searle, J.R. (1972), Les actes de langage. Essai de philosophie du langage, Hermann, Paris

Searle, J.R. (1998), La construction de la réalité sociale, Gallimard, Paris

Trichet, J-C. (2005), “Communication, Transparency and the ECB’s Monetary Policy”, Keynote speech at the New Year's reception of the International Club of Frankfurt Economic Journalists, Francfort, 24 January.

Velthuis, O. (2015), « Making monetary markets transparent: the European Central Bank's communication policy and its interactions with the media ", Economy and Society, 44:2, pp. 316-340

Volcker, P. (2009), « The triumph of central banking? ", The Per Jacobsson Lecture, Washington. Woodford, M. (2001), « Monetary Policy in the Information Economy » in Economic Policy for the Information Economy, Kansas City: Federal Reserve Bank of Kansas City, pp. 297-370.

Woodford, M. (2003), Interest and Prices: Foundations of a Theory of Monetary Policy, Princeton University Press.

Woodford, M. (2005), «Central-bank communication and policy effectiveness », NBER Working Paper, vol. 11898, National Bureau of Economic Research, Cambridge MA.

Zadovny, M., Ginther, (2005), D.K. « Does the Beige Book Move Financial Markets? », Southern

Economic Journal, 2005, 72 (1), pp. 138-151. 


\section{NOTES}

1. Searle propose un exemple à la jonction de l'économie et du droit : «Même l'énoncé que l'on peut trouver sur le billet de vingt dollars, bien que ne contenant pas de verbes performatifs, est une déclaration. Il dit : «Ce billet est le cours légal pour toutes dettes, publiques et privées.» Mais cette énonciation n'est pas une affirmation empirique.» (Searle 1998, p. 78). Par cette mention imprimée, le Trésor Public -considéré comme légitime- transforme le billet vert en moyen de paiement non contestable.

2. "The issue that needs to be tackled in relation to economies and economics is not just about "knowing" the world, accurately or not. It is also about producing it. ", MacKenzie, Muniesa, Siu, 2007, p. 2. Ainsi l' option pricing theory de Black-Scholes-Merton a permis aux marchés dérivés de connaitre un essor phénoménal, voir McKenzie, 2007.

3. Les Banques Centrales, selon Callon et Muniesa font partie de ces « instances 'd'économisation' du monde ", Callon, Muniesa, 2008, p. 2.

4. Pour ce faire, nous employons une méthode de traitement textuel, utilisée par Saddique et al. (2013) sur les éditoriaux des Beige Book de la FED : il s'agit de recenser le nombre de mots de chaque éditorial du Bulletin mensuel puis, suivant leur connotation positive ou négative, des indicateurs d'optimisme ou de pessimisme sont créés. On mesure ainsi les inflexions langagières de l'institut d'émission.

5. L'expression est utilisée par Lebaron dans son ouvrage de 2010, p. 74.

6. "Within our mandate, the ECB is ready to do whatever it takes to preserve the euro. And believe me, it will be enough." European Central Bank (2012), "Verbatim of the remarks made by Mario Draghi Speech by Mario Draghi, President of the European Central Bank at the Global Investment Conference in London, 26 July2012", www.ecb.int/press/key/date/2012/html/sp120726.en.html

7. «la production linguistique est inévitablement affectée par l'anticipation des sanctions du marché », Bourdieu, 2001, p. 114.

8. Keynes: "[...] is it a practice of the Bank of England never to explain what its policy is?"

Harvey: "Well, I think it has been our practice to leave our actions to explain our policy."

Keynes: "Or the reasons for its policy?"

Harvey: "It is a dangerous thing to start to give reasons.", cite par Issing, 2005, p. 1

9. "Central Banking... thrives on a pervasive impression that [it]... is an esoteric art. Access to this art and its proper execution is confined to the initiated elite. The esoteric nature of the art is moreover revealed by an inherent impossibility to articulate its insights in explicit and intelligible words and sentences.", Brunner, 1981, p. 5.

10. Selon Kydland et Prescott, « les autorités politiques issues du processus électoral [...], plus soucieuses de satisfaire immédiatement leur électorat que d'assurer le bien-être économique à moyen et long terme de leurs administrés et de leurs descendants, auraient en effet tendance à laisser augmenter l'inflation en échange d'un surcroît de bien-être temporaire et factice.", Lebaron, 2006, p. 21.

11. "Autonomous central banks thus act as a precommitment device which may help to make noninflationary policies more credible and less costly", Backus, Drifill, 1985, p. 537.

12. Toutefois, l'ancien gouverneur de la Fed, Alan Greenspan se décrivait lui-même comme émettant un marmonnement sans grande cohérence pour affirmer que si on l'avait compris, c'est qu'il s'était mal exprimé... "Since becoming a central banker, I have learned to mumble with great incoherence. If I seem unduly clear to you, you must have misunderstood what I said » (discours au comité du Sénat, 1987 repris dans le Guardian Weekly, du 4 novembre 2005).

13. "There is every reason to believe that [communication] this has been beneficial, not only from the point of view of reducing the uncertainty with which traders and other economic decisionmakers must 
contend, but also from that of enhancing the accuracy with which the FOMC is able to achieve the effects on the economy that it desires, by keeping the expectations of market participants more closely synchronized with its own. ", Woodford, 2005, p. 56.

14. "Financial markets evidence indeed indicates that we as a central bank have managed to be understood by market professionals. (...) One of the main goal of a responsible central bank now is to have a reliable communication guiding expectations in a rapidly changing environment », Trichet, 2005.

15. «Une interprétation correcte par le marché des décisions de politique monétaire prises par la Banque centrale réduit la volatilité du taux d'intérêt» et, par conséquent, "une bonne compréhension de la politique monétaire permet aux agents privés de mieux gérer et couvrir leurs risques, ce qui peut contribuer à réduire l'incertitude du marché et à améliorer le bien-être économique ", BCE, 2002, p. 62.

16. "Lorsque le plancher zéro est atteint, les agents sont enclins à anticiper une remontée des taux ; ils en déduisent que le prix des titres va continuer de baisser et la crise s'approfondir ; ils anticipent une augmentation du risque de défaillance et la persistance de la déflation. Face à cela, l'objectif de la communication des Banques centrales est de rassurer pour orienter les anticipations des agents vers la sortie de crise. Si les Banques centrales annoncent que les taux resteront au plancher tant que la crise n'aura pas été jugulée, les agents anticiperont le maintien de taux courts bas (au lieu de leur hausse), un accès facilité à la liquidité (et donc une réduction des primes de risque) et, à la condition que la Banque centrale leur apparaisse suffisamment crédible et déterminée, la fin de la déflation. » Couppey-Soubeyran, 2012, p. 54.

17. "Central bank transparency could be defined as the absence of asymmetric information between monetary policy makers and other economic agents. This means that it reduces uncertainty and this is often believed to be beneficial », Geraats 2002, p. 533.

18. Voir Carré (2005) et Carré, Le Héron (2006).

19. En accord avec les théories mentionnées plus haut, la cible du grand public est d'une importance cruciale pour la Banque dans la mesure où les anticipations d'inflation pourraient alimenter l'évolution effective de l'inflation (que ce soit à travers les revendications salariales, les décisions d'épargne, la consommation...). Toutefois, le public réagit rarement à la communication directe, de sorte que cette cible ne peut être touchée que par une communication indirecte à travers les médias.

20. On peut citer, d'une part, les travaux de Rosa et Verga (2005) (de janvier 2002 à décembre 2004), de Berger, De Haan et al. (2011) (de janvier 1999 à décembre 2004), de Rosa et Verga (2006, 2007), d'Heinemann et Ullrich (2007) qui utilisent la déclaration introductive du président de la BCE durant la conférence de presse. D'autre part, les études de Gerlach, (2004, de janvier 1999 à juin 2004, et 2007) et de Bennani et Farvaque (2014, de 1999 à 2005) utilisent l'éditorial du Bulletin mensuel de la BCE.

21. Un autre type de problème surgit lors de la divulgation d'informations privilégiées, comme cela fut le cas le 18 mai 2015 : lors d'une rencontre entre un membre de la BCE (Benoit Coeuré) et des investisseurs (banques et hedge funds), une information sensible fut révélée, à savoir que la $\mathrm{BCE}$ allait accélérer son rachat d'actifs. Or, aucune information ne doit donner un avantage à un groupe d'investisseurs. L'information doit, en effet, être accessible à tous au même moment. Suite à cet «incident », la BCE a décidé de ne plus diffuser aux médias de copie de ses discours sous embargo, de sorte que l'information arrivera à tous au même moment.

22. Dictionnaire généralement utilisé dans ce type d'analyse, même s'il n'est pas conçu à l'origine par des économistes ou des financiers: "A commonly used source for word classifications is the Harvard Psychosociological Dictionary, specifically, the Harvard-IV-4 TagNeg (H4N) file. One positive feature of this list for research is that its composition is beyond the control of the researcher. That is, the researcher cannot pick and choose which words have negative implications ", Loughran, Mc Donald, 2011, p. 35. 
23. Une analyse granulaire du corpus modifie le nombre de mots retenus; en sélectionnant une série de mots, le sens peut changer.

24. Bourdieu, à partir d'un article du Monde qualifiant le président de la Bundesbank de « grand prêtre du Deutschemark » précisait « car c'est bien de religion qu'il s'agit », Bourdieu, 1996.

25. "Within our mandate, the ECB is ready to do whatever it takes to preserve the euro. And believe me, it will be enough" European Central Bank (2012), "Verbatim of the remarks made by Mario Draghi Speech by Mario Draghi, President of the European Central Bank at the Global Investment Conference in London, 26 July 2012", www.ecb.int/press/key/date/2012/html/sp120726.en.html

26. Désormais la crédibilité serait assurée par la monétisation de la dette... Ainsi la monétisation de plus de 1000 milliards d'euros qui devrait constituer une hérésie dans le cadre de la théorie standard est devenue une nécessité naturelle. La crise a conduit à une volte-face dans la pratique de la politique monétaire qui aurait dû entraîner une remise en cause de la doctrine dominante. Il n'en a rien été ce qui montre bien que la doctrine n'est opératoire, non pas en tant qu'instrument de politique économique, mais tant qu'outil d'économie politique.

27. Dans les cas de «miracles monétaires » analysés par A. Orléan (Allemagne en 1923, France en 1926), c'est la population qui est concernée, pas uniquement les acquéreurs de titres. La première partie de la citation suivante précise ce qui peut être qualifié de miraculeux (" pur mouvement d'adhésion collective ») et le caractère monétaire de la transformation (« réunir la population ») : «Dans les deux épisodes, le retournement ne trouve pas sa source dans les mesures de politique économique. Il s'agit d'un pur mouvement d'adhésion collective, de croyance mimétique de tout le groupe, conforme à notre modèle. Cela explique sa brutalité et son caractère " miraculeux ». Sa réussite tient pour partie au rôle que jouent certains symboles forts, aptes à réunir la population autour de la nouvelle norme monétaire. ", Orléan, 2011, p. 225.

28. Brisset, 2014 , p. 219 : "performer c'est mettre en place une nouvelle convention sociale définie comme une représentation commune du monde. »

29. «[...] le traité de Maastricht a conféré à la BCE un statut véritablement extraordinaire. Contrairement à toutes les autres banques centrales du monde, sa légitimité n'est fondée sur aucune souveraineté politique. Elle est véritablement déterritorialisée. ", Aglietta, 2011, p. 11.

30. Rogoff évoque la création d' " une bulle autour des prises de position et des décisions des banques centrales, qui exagère très largement leur importance économique. ", Rogoff, 2014.

31. C'est la raison pour laquelle Bibow (2010) qualifie de "douteuse" cette victoire de l'argumentaire en faveur de l'indépendance des Banques Centrales. «Au sujet des banques centrales, deux perspectives assez contradictoires coexistent aujourd'hui dans les discours publics. Pour la théorie économique, la banque centrale est une unité de décision rationnelle et stratégique qui se situe dans un cadre juridique, se fixe des objectifs et déploie divers objectifs intermédiaires et instruments pour les atteindre. Pour beaucoup de commentateurs, à l'opposé, le succès d'une banque centrale semble très directement lié à la personnalité singulière de son gouverneur. » Lebaron, 2006, p. 38.

\section{RÉSUMÉS}

Cet article, vise à comprendre pourquoi le discours des banquiers centraux a été paré d'un poids significatif depuis quelques années. À partir des éléments mis en avant dans la théorie économique standard, d'une part, et la sociologie, d'autre part, nous étudions les dispositifs qui ont permis cette transformation et analysons certains actes de langage. 
Nous revenons dans un premier temps sur le contexte théorique qui a favorisé la mise en exergue de la parole des banquiers centraux pour les économistes. La première section résume les arguments en faveur de la communication de la Banque centrale développés par les Nouveaux Classiques puis les Nouveaux Keynésiens. Nous décrivons ensuite rapidement les divers canaux de transmission.

Dans une deuxième partie, nous revenons sur les «actes de langage » de la BCE en étudiant les pratiques et les outils employés. Nous étudions ensuite la tonalité de cette communication : à partir des mots utilisés dans les éditoriaux du bulletin mensuel de l'institution (et leur connotation positive ou négative), on crée par un traitement linguistique des indicateurs d'optimisme ou de pessimisme. Dans une troisième partie, nous interrogeons les rapports qui s'établissent entre le locuteur et les récepteurs des messages. Cette question nous amène à discuter la distinction entre effets performatifs (production ou transformation de la réalité) et perlocutoires (action sur les représentations). Nous revenons enfin sur les raisons de cette mise en exergue de la parole du banquier central: le locuteur n'est-il pas le porte-parole des opérateurs sur les marchés financiers?

This paper aims at understanding why the central banks' talks took a significant weight recently. By using some tools, put forward in the economic theory, on the one hand, and on sociology, on the other hand, we study the devices permitting this transformation and we analyse some "acts of speech". First, we describe how the theoretical context led to highlight the speeches of central bankers for the economists. The first section summarizes the arguments in favour of the communication of the central Bank developed by the New Classicals and the New Keynesian Economics. Then, we describe the various transmission channels. Second, we come back on the "acts of speech" from the ECB by studying its practices. We analyse the tone of this communication. We create indicators of optimism or pessimism through a text mining approach based on the words (and their positive or negative connotation) used in the editorials of the ECB monthly bulletin. Finally, we question the relationships established between the speaker and the receivers of those messages. This question brings us to discuss the distinction between performative effects (production of the reality) and perlocution (action on the representations). We conclude on the reasons of the communicational turn of central bankers, namely that the speaker can be seen as the spokes-man of financial markets' operators.

\section{INDEX}

Mots-clés : communication, BCE (Banque centrale européenne), publics, performativité

Keywords : communication, ECB (European Central Bank), public, performativity

Code JEL B22 - Macroeconomics, E52 - Monetary Policy, E58 - Central Bank and Their Policies

\section{AUTEURS}

\section{MICKAËL CLÉVENOT}

LEDi, université de Bourgogne

\section{LUDOVIC DESMEDT}

LEDi, université de Bourgogne

\section{MATTHIEU LLORCA}

CEPN, université Paris 13 